\title{
Detailed performance evaluation of a new 20-inch photomultiplier tube with a Box and Line dynode
}

\section{Yuji Okajima*}

Department of Physics, Tokyo Institute of Technology

E-mail: okajima@hep.phys.titech.ac.jp

Yasuhiro Nishimura ${ }^{a}$, Ryosuke Akutsu $^{a}$, Yusuke Suda $^{b}$, Miao Jiang $^{c}$, Seiko Hirota ${ }^{c}$, Daisuke Fukuda $^{d}$, Masahiro Kuze $^{e}$, Masaki Ishitsuka ${ }^{e}$, Masayuki Nakahata ${ }^{a}$, Masato Shiozawa $^{a}$, Yoshinari Hayato ${ }^{a}$, Shoei Nakayama ${ }^{a}$, Hidekazu Tanaka ${ }^{a}$, Masashi Yokoyama $^{b}$, Tsuyoshi Nakaya ${ }^{c}$, Akihiro Minamino $^{c}$, Akimichi Taketa $^{f}$, Yoshihiko Kawai $^{g}$, Takayuki Ohmura ${ }^{g}$, Masatoshi Suzuki ${ }^{g}$

${ }^{a}$ Institute for Cosmic Ray Research, University of Tokyo

${ }^{b}$ Department of Physics, University of Tokyo

${ }^{c}$ Department of Physics, Kyoto University

${ }^{d}$ Department of Physics, Okayama University

e Department of Physics, Tokyo Institute of Technology

${ }^{f}$ Earthquake Research Institute, University of Tokyo

${ }^{g}$ Hamamatsu Photonics K.K.

For Hyper-Kamiokande, which is a future planned experiment using a 1 Mton water Cherenkov detector, several types of new photodetectors are under development. Box \& Line photomultiplier tube is the leading candidate with high resolution and efficiency. By the improvement on quantum efficiency and photoelectron collection efficiency, the Cherenkov photon detection efficiency is twice as high as that of the photomultiplier tube used for Super-Kamiokande. The detailed performance of the Box \& Line photomultiplier tube was evaluated to check its appropriateness for Hyper-Kamiokande, and it was confirmed to be sufficiently good. At single photoelectron level, a high resolution of $35 \% \sigma / \mu$ in charge and $4.1 \mathrm{~ns}$ at FWHM in transit time was confirmed, superior to $53 \%$ and $7.3 \mathrm{~ns}$, respectively, in case of the Super-K photomultiplier tube. The linearity range within 340 photoelectrons and high rate tolerance up to $87 \mathrm{MHz}$ at single photoelectron are evaluated. The dark rate at room temperature is about $10 \mathrm{kHz}$ typically.

International Conference on New Photo-detectors PhotoDet2015,

6-9 July 2015

Moscow, Troitsk, Russia

${ }^{*}$ Speaker. 


\section{Hyper-Kamiokande project}

The Hyper-Kamiokande project [1] is a successor of a neutrino experiment, Super-Kamiokande, which is a successful experiment with rich physics topics such as the discovery of neutrino oscillation and search for nucleon decay. It has one-megaton water for neutrino detection as a Cherenkov detector, which is 20 times larger than Super-Kamiokande. The physics targets are measurement of neutrino oscillation parameters, proton decay, diverse astrophysics studies and so on. Much more 20 inch $\phi$ photodetectors than Super-Kamiokande (Hyper-K plan: 99000 tubes, Super-K: 11129 tubes) will be used in the inner detector to cover $20 \%$ of the detector wall. The coverage is half of the coverage of Super-Kamiokande, and this makes the performance of Hyper-Kamiokande worse. Therefore, the photodetectors in Hyper-Kamiokande are required to be low cost and high performance, which means not only high resolution and detection efficiency, but also a low dark rate for measurement of low energy particles like solar neutrino and stable gain for a possible high input rate signals from supernovae.

\section{Box \& Line PMT}

20 inch $\phi$ R12860 photomultiplier tube (PMT) by Hamamatsu Photonics K.K. (Hamamatsu) is a candidate photodetector for Hyper-Kamiokande, which has Box and Line type dynodes. The dynode shape is different from Hamamatsu R3600 (20 inch $\phi$ PMT for Super-Kamiokande), as shown in Fig.1. Because of the dynode shape improvement, Box \& Line PMT has uniform path length and high collection efficiency of photoelectrons. Uniform path length provides the PMT with a good transit time spread (TTS). Because of the less complicated structure than R3600, Box \& Line PMT is expected to be produced at a low cost. Currently, a Box \& Line PMT Hamamatsu R7250 is used for the KamLAND experiment which has a 17 inch $\phi$ effective region with a 20 inch $\phi$ bulb. The new Box \& Line PMT for Hyper-Kamiokande was developed to improve the collection efficiency uniformly in a whole 20 inch area. For this purpose, the following improvements were pursued:

- Optimize curvature and dynode alignment to improve photoelectron collection efficiency.

- A focusing electrode was added.

- The detection area of first dynode was enlarged.

New Box \& Line PMT has high collection efficiency (CE), 95\% (85\%) in $\phi 460 \mathrm{~mm}$ ( $\phi 498$ $\mathrm{mm}$ ) region of the photocathode surface. In addition, its photocathode has been improved and the peak quantum efficiency (QE) became about 30\%, which is higher than R3600 (about 22\%), as shown in Fig.2. Tab.1 shows the summary of photodetector efficiencies.

\section{Performance evaluation}

\subsection{Pulse shape}

The pulse shapes of R3600 and the Box \& Line PMT with two types of the bleeder circuits are measured. One bleeder circuit has a fast time response, while the other has a ringing countermeasure structure. As shown in Fig.3 and Tab.2, the Box \& Line PMT produces faster pulse 


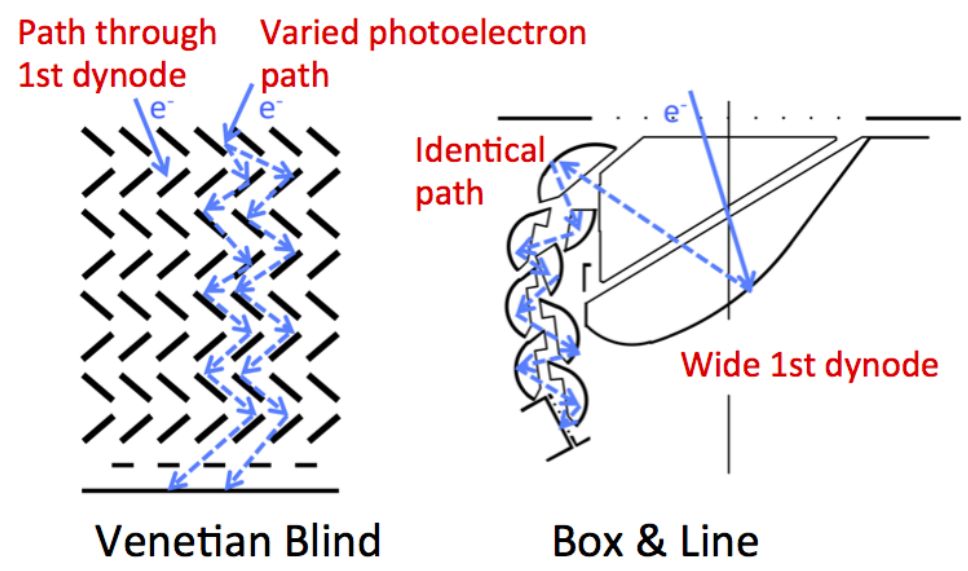

Figure 1: Structure of Venetian Blind (Left) and Box \& Line dynode systems (Right).

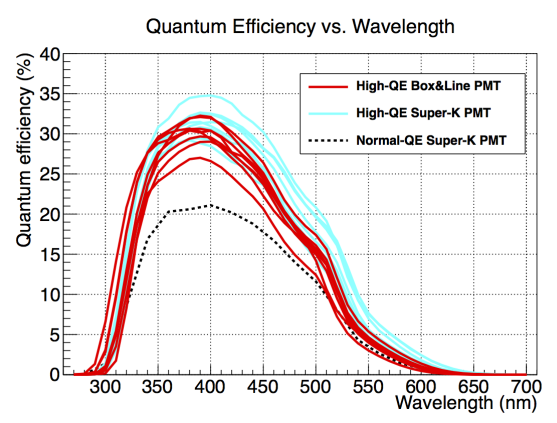

\begin{tabular}{ccc}
\hline & R3600 & Box \& Line PMT \\
\hline Peak quantum efficiency & $\sim 22 \%$ & $\sim 30 \%$ \\
\hline Collection efficiency for & $67 \%(61 \%)$ & $95 \%(85 \%)$ \\
$\phi 460 \mathrm{~mm}(\phi 498 \mathrm{~mm})$ & & \\
\hline Total efficiency for & $\sim 15 \%(\sim 13 \%)$ & $\sim 29 \%(\sim 26 \%)$ \\
$\phi 460 \mathrm{~mm}(\phi 498 \mathrm{~mm})$ & & \\
$(\mathrm{QE} \times \mathrm{CE})$ & & \\
\hline
\end{tabular}

Figure 2: QE spectrum of high QE Box \& Line PMT, high QE R3600 and normal QE Table 1: Efficiency of sensors. R3600.

than R3600. Also, one of the two circuit reduces the ringing effect and therefore it was used for evaluation in this paper.

\subsection{Gain}

The gain as a function of HV is shown in Fig.4 (Left) for several Box \& Line PMT samples. Box \& Line PMT has comparable gain with R3600, and the nominal HV is determined at $\sim 10^{7}$ gain, which is the same as R3600 in Super-Kamiokande.

\subsection{Dark rate}

The dark rate of PMTs tends to decrease at low temperatures and it takes long time to stabilize. To verify that the Box \& Line PMT has enough good performance for the Hyper-Kamiokande detector, it is necessary to evaluate performance in the same environment as that in the Hyper-K detector. As a simple verification test of up-to-date version Box \& Line PMT, the dark rate was measured for 20 days, in the $14^{\circ} \mathrm{C}$ room (almost the same temperature as Super-K water tank). As a result, the dark rate was reduced from $11 \mathrm{kHz}$ (measurement after 5 hours from $\mathrm{HV}$ on) to $9 \mathrm{kHz}$ 


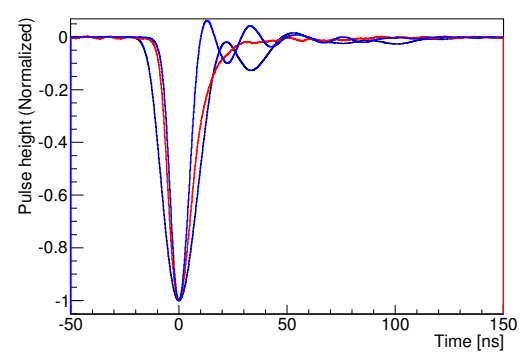

Figure 3: Pulse shape of PMTs. Dark and blight blue, and red lines show pulse shape of R3600 and the Box \& Line PMT with faster bleeder and smooth bleeder circuits, respectively.

\begin{tabular}{cccc}
\hline & $\begin{array}{c}\text { Rise time } \\
(10 \%-90 \%)\end{array}$ & $\begin{array}{c}\text { Fall time } \\
(10 \%-90 \%)\end{array}$ & $\begin{array}{c}\text { Pulse width } \\
(\text { FWHM })\end{array}$ \\
\hline R3600 & $10.6 \mathrm{~ns}$ & $13.2 \mathrm{~ns}$ & $18.8 \mathrm{~ns}$ \\
\hline $\begin{array}{c}\text { Box \& Line PMT } \\
\text { (Fast bleeder) }\end{array}$ & $6.2 \mathrm{~ns}$ & $6.3 \mathrm{~ns}$ & $10.0 \mathrm{~ns}$ \\
\hline $\begin{array}{c}\text { Box \& Line PMT } \\
\text { (Smooth bleeder) }\end{array}$ & $6.8 \mathrm{~ns}$ & $15.2 \mathrm{~ns}$ & $13.2 \mathrm{~ns}$ \\
\hline
\end{tabular}

Table 2: Pulse shape. Rise and fall times are defined as the interval between the time when voltage becomes $10 \%$ and $90 \%$ height of pulse peak. Pulse width is defined as FWHM of the pulse.
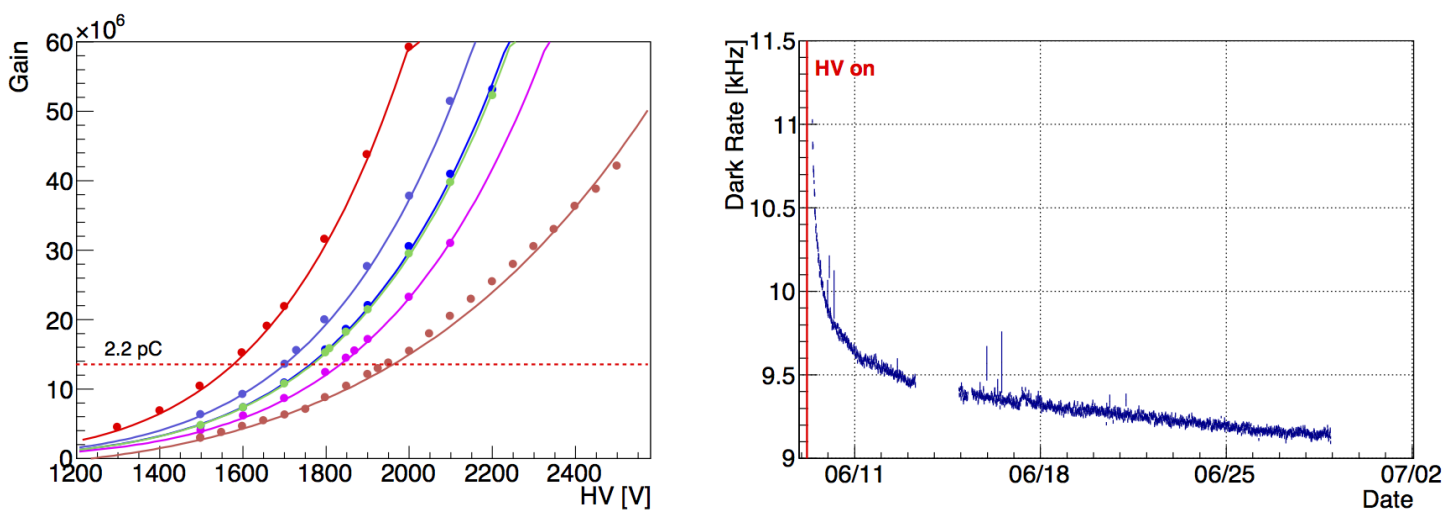

Figure 4: Box \& Line PMT performance (Left: Gain as a function of HV by several samples shown in different colors, where the gain of $2.2 \mathrm{pC}$ is the nominal HV of Super-Kamiokande. Right: Stabilization of dark rate as a function of measured date, measured at $2 \mathrm{mV}$ threshold at $14{ }^{\circ} \mathrm{C}$.)

(20 days after $\mathrm{HV}$ on). Considering the twice-higher photoelectron detection efficiency, the dark rate got comparable with that of $\mathrm{R} 3600(4.2 \mathrm{kHz})$.

\subsection{Single photoelectron response}

The charge and transit time distributions for single photoelectron events were measured as shown in Fig.5 and Tab.3. TTS is evaluated by fitting the distribution with an exponentially modified Gaussian function (EMG, Eq.3.1).

$$
\begin{aligned}
f(x ; \mu, \sigma, \lambda) & =\frac{\lambda}{2} e^{\frac{\lambda}{2}\left(2 \mu+\lambda \sigma^{2}-2 x\right)} \operatorname{erfc}\left(\frac{\mu+\lambda \sigma^{2}-x}{\sqrt{2} \sigma}\right) \\
\operatorname{erfc}(x) & =\frac{2}{\sqrt{\pi}} \int_{x}^{\infty} e^{-t^{2}} d t
\end{aligned}
$$

The $\sigma$ and FWHM of EMG function were evaluated as TTS. This result confirmed that the Box \& Line PMT has better charge and TTS at single photoelectron level. 

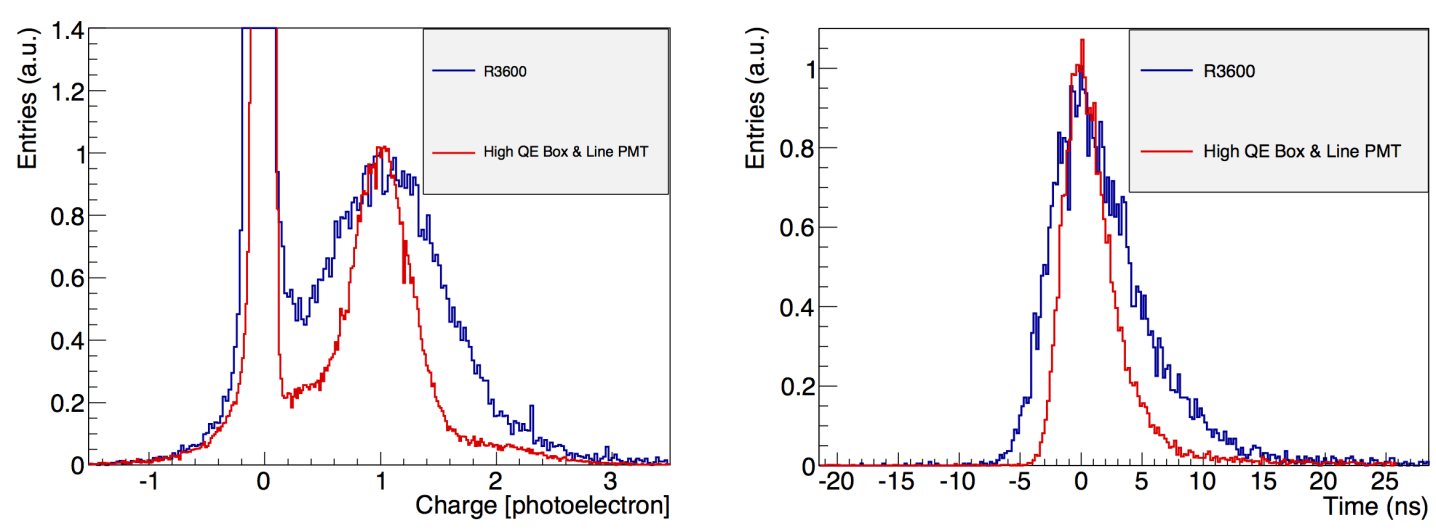

Figure 5: Single photoelectron response. (Left: Charge distribution, Right: Transit time distribution)

\begin{tabular}{|c|c|c|c|c|}
\hline & Charge resolution $(\sigma / \mu)$ & Peak/Valley Ratio & \multicolumn{2}{|c|}{ TTS } \\
\cline { 4 - 5 } & & & $\sigma$ & FWHM \\
\hline R3600 & $53 \%$ & 2.2 & $2.1 \mathrm{~ns}$ & $7.3 \mathrm{~ns}$ \\
\hline Box \& Line PMT & $35 \%$ & 4.3 & $1.1 \mathrm{~ns}$ & $4.1 \mathrm{~ns}$ \\
\hline
\end{tabular}

Table 3: Response at single photoelectron.

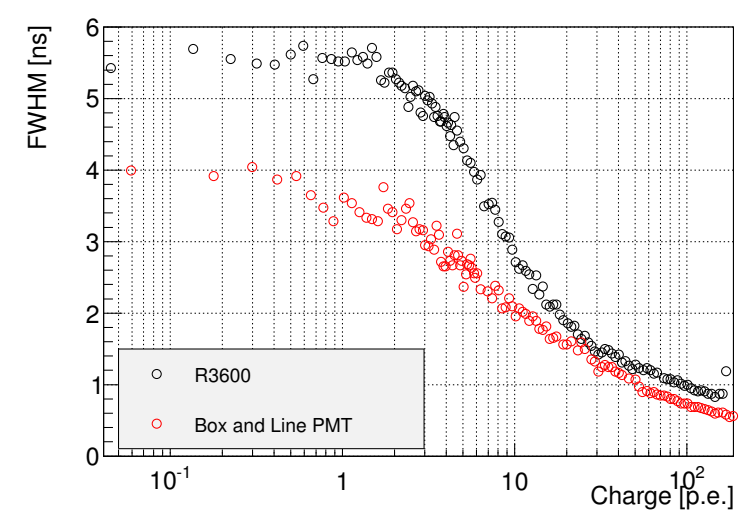

Figure 6: TTS of PMTs. (FWHM)

\subsection{Timing resolution}

After the correction of charge dependence of transit time, the TTS is parameterized with EMG in various charge range. Fig.6 shows the TTS evaluated at FWHM in a wide input range. From this result, the Box \& Line PMT shows the better TTS in the whole region (2/3 FWHM of R3600 at single photoelectron level). 

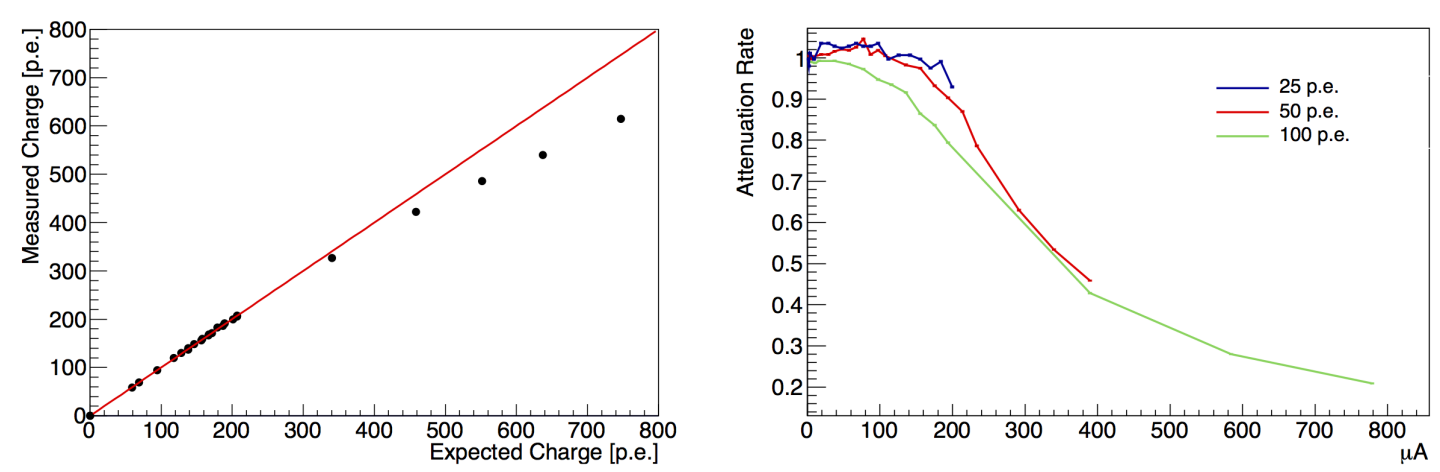

Figure 7: Left: Linearity of the Box \& Line PMT. Right: Rate tolerance as a function of charge current.

\subsection{Gain linearity}

High intensity signal can saturate the response of a PMT, due to a limit of the current in the bleeder circuit and the large number of electrons in dynodes. The dynamic range for the linear output response was evaluated using two light sources, for comparison of a sum of each output by individual light emissions and a coincident light of two with varying the intensity of only one light source. In this measurement, the gain was set to $2.0 \mathrm{pC}$ for a single photoelectron. Fig.7 (Left) shows the result. Our measurement showed that the Box \& Line PMT is kept the linearity up to 340 p.e. with $5 \%$ gain drop.

\subsection{Rate tolerance}

In order to measure very frequent signals like those, for example, possible from supernovae, the output stability of the Box \& Line PMT against high frequency light pulses was checked. In order to evaluate the tolerance, PMT was exposed toadied light from two laser diodes with the same intensity. One of the two laser diodes makes $100 \mathrm{~Hz}$ signal pulses to monitor, and the other laser diode makes various rate off-time laser pulses. The intensities of these two diodes were set to 25,50 , and 100 photoelectron levels. The rate tolerance was evaluated not only as a function of frequency, but also as a function of current. The current was defined as a product of pulse frequency and charge of a single pulse. The result of evaluation as a function of current is shown in Fig.7 (Right). It was shown that gain drops by less than 5\% up to $170 \mu \mathrm{A}$ for 50 photoelectrons. By considering the current, the gain is expected to be stable up to $87 \mathrm{MHz}$ single photoelectron pulse injection. The supernova residing at the galactic center and that of Betelgeuse are expected to provide a low-energy neutrino maximum rate of $200 \mathrm{kHz}$ and $50 \mathrm{MHz}$ respectively, with hits less than a few photoelectrons for each PMT.

\subsection{Gain recovery}

In order to identify events with two consecutive signals in Hyper-Kamiokande, such as $\mu$ decay, a quick gain recovery is important. Two continuous signals are emitted from two laser diodes whose intensities are fixed to 140 p.e. level. By comparing the delayed signal charge with 


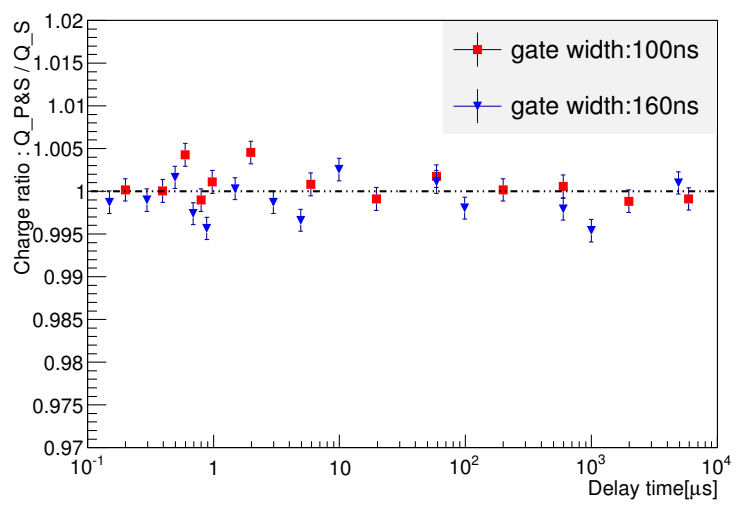

Figure 8: Charge ratio of signals emitted after primary signal emission to the same intensity signals without primary signal as a function of the interval between primary signal and measured signal. The error bar shows the statistical error.

and without primary signal, the gain was evaluated. Fig. 8 shows that no significant changes were observed, and stable within $0.5 \%$, which is comparable with statistical fluctuation.

\section{Summary}

In order to improve the performance and to lower the cost, new photodetectors were developed. In this article, we evaluated the Box \& Line PMT, which is a candidate of photodetectors for HyperKamiokande. The pulse is faster (FWHM with smooth bleeder: $13.2 \mathrm{~ns}$ ) than that of R3600. In addition, the Box \& Line PMT shows better charge resolution $(\sigma / \mu: 35 \%)$ and TTS (FWHM: 4.1 ns) at single photoelectron. The dark rate of the Box \& Line PMT is $10 \mathrm{kHz}$ on average, measured at the single photoelectron level. The rate tolerance and linearity keeps until $170 \mu \mathrm{A}$ (corresponds to $87 \mathrm{MHz}$ single photoelectron level) and 340-photoelectron level, respectively. In gain recovery measurement, there were no significant variations observed. From these results, it was shown that the Box \& Line PMT has better performance than the current PMT used in Super-Kamiokande and it is sufficient for Hyper-Kamiokande.

\section{References}

[1] Hyper-Kamiokande working group "Letter of Intent: The Hyper-Kamiokande Experiment Detector Design and Physics Potential —", arXiv:1109.3262 